\title{
An approximate solution to the optimal coordination problem for autonomous vehicles at intersections
}

\author{
Robert Hult, Gabriel R. Campos, Paolo Falcone, Henk Wymeersch
}

\begin{abstract}
In this paper, we address the problem of optimal and safe coordination of autonomous vehicles through a traffic intersection. We state the problem as a finite time, constrained optimal control problem, a combinatorial optimization problem that is difficult to solve in real-time. A low complexity computational scheme is proposed, based on a hierarchical decomposition of the original optimal control formulation, where a central coordination problem is solved together with a number of local optimal control problems for each vehicle. We show how the proposed decomposition allows a reduction of the complexity of the central problem, provided that approximated parametric solutions of the local problems are available beforehand. We derive conditions for the construction of the parametric approximations and demonstrate the method with a numerical example.
\end{abstract}

\section{INTRODUCTION}

While autonomous vehicles today are mere demonstrators of the technological capabilities and achievements of car manufacturers and universities, they are likely to penetrate the market on a broad scale in the future. Together with widespread use of vehicle-to-vehicle (V2V) communication this will transform the road traffic system and enable large improvements in terms of safety, energy efficiency and infrastructure utilization [1]. A particularly problematic subset of the scenarios in the traffic system are intersections, in which a disproportionally large proportion of accidents, injuries and fatalities occur, and where a large part of inefficiencies originate [2]. It is therefore natural to investigate coordination algorithms for autonomous vehicles at intersections and how this technology could be exploited to alleviate these issues. In particular, scenarios where all vehicles are autonomous and communicating offer the possibility to remove current coordination mechanisms (e.g., traffic lights, signs and rules) and solely rely on cooperative coordination among the involved vehicles. Besides safe operation, optimization with respect to objectives like overall energy efficiency are then possible by, e.g., slowing down lighter vehicles in favour of heavier ones [1] as soon as they are within a reliable wireless communication range [3].

Several algorithms have been presented that address the coordination problem at intersections for fully autonomous vehicles. Commonly, the presence of a central decision

*This work is supported by The Swedish Research Council under Grant No. 2012-4038, Chalmers' Area of Advance in Transportation, SAFER, the Department of Signals and Systems (S2) and by the European Research Council under Grant No. 258418 (COOPNET).

The authors are with the Department of Signals and Systems, Chalmers University of Technology, Gothenburg, Sweden. Email: \{robert.hult, gabriel.campos, paolo.falcone, henkw\}@chalmers.se maker is assumed, which manage time-space reservations in the intersection to avoid collisions [4-8]. However, several decentralized schemes have also been considered, e.g., as in [9], [10], based on reachability analysis and sequential decision making, or in [11] based on event driven interaction protocols. Most of the existing work place heavy emphasis on safety and collision avoidance, and schemes that are designed to simultaneously address efficiency are rare.

In this paper, we formulate and study the intersection coordination problem for autonomous vehicles using a finite time, constrained optimal control formalism, where the global (intersection-wide) objective is to optimize a sum of local costs. The formulation results in the prohibitively hard combinatorial problem of choosing the order in which the vehicles cross the intersection, constrained by the vehicle dynamics and physical limitations.

The main contribution of this paper is a decomposition scheme that gives an approximate solution to the original optimal control problem with significantly lower demands on computational capabilities and information exchange. In particular, the combinatorial part of the problem (the vehicle crossing order) is first separated from the problem of finding the appropriate control inputs, and then solved approximately, giving guaranteed collision free intersection occupancy time slots that are feasible under the vehicle dynamics and physical constraints. The control policies are thereafter found by solving one optimal control problem for each vehicle, constrained so that occupancy of the intersection is allowed only within the allocated time slot.

\section{Problem Statement and Formulation}

We consider a scenario where $N \in \mathbb{Z}_{+}$vehicles approach a traffic intersection along predefined paths, as visualized in Fig. 1a. There is only one vehicle per path and the traffic intersection is therefore the area where two or more paths paths intersect. The coordination is then the problem of controlling the motion of each vehicle along its path such that that access to the intersection area is mutually exclusive. The motion dynamics along the path of the $i$-th vehicle are described by

$$
\begin{aligned}
\dot{x}_{i}(t) & =A_{i} x_{i}(t)+B_{i} u_{i}(t), \\
y_{i}(t) & =C_{i} x_{i}(t),
\end{aligned}
$$

where $x_{i}(t) \in \mathbb{R}^{n_{i}}$ and $u_{i}(t) \in \mathbb{R}^{m_{i}}$ are the state and control input vectors and the scalar output $y_{i}(t)$ is the position along the path. The pair $\left(A_{i}, B_{i}\right)$ is assumed controllable and the state and input trajectories are constrained by the 


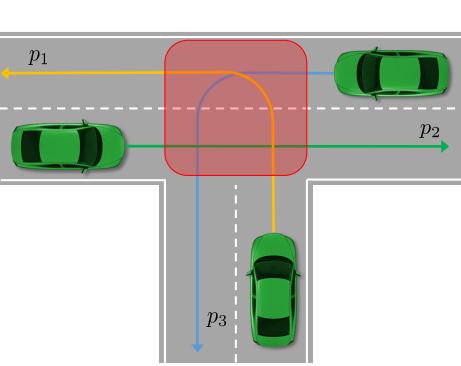

(a)

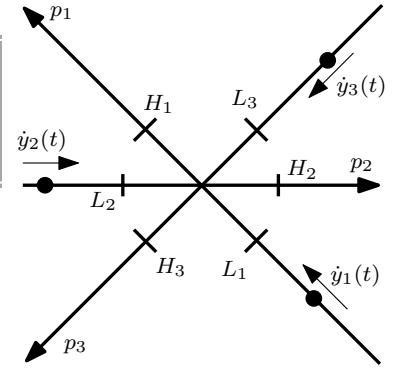

(b)
Fig. 1: Illustration of the considered scenario: (a) A road intersection scenario with predefined paths $p_{1}, p_{2}, p_{3}$. The region where collisions can occur is marked in red, (b) The abstraction of the intersection used in modelling.

linear inequalities

$$
\begin{aligned}
& G_{i} x(t) \leq b_{i}, \forall t, \\
& F_{i} u(t) \leq d_{i}, \quad \forall t,
\end{aligned}
$$

with $G_{i} \in \mathbb{R}^{k_{i} \times n_{i}}, b_{i} \in \mathbb{R}^{k_{i}}, F_{i} \in \mathbb{R}^{q_{i} \times m_{i}}, d_{i} \in \mathbb{R}^{q_{i}}$, arising from, e.g., actuator limitations and design requirements. Additionally, for technical reasons we only consider strongly output monotone systems, i.e., systems satisfying

$$
\dot{y}_{i}(t)=C_{i} \dot{x}_{i}(t) \geq \varepsilon, \forall t,
$$

for some $\varepsilon>0$. Note that (3) implies that a vehicle can neither reverse nor stop at any time, but that arbitrarily small $\varepsilon$ are possible and therefore arbitrarily low speeds. For brevity, we denote by $D_{i}\left(x_{0, i}\right)$ the set of solutions $\left(x_{i}(t), u_{i}(t)\right)$ to (1) with initial condition $x_{i}(0)=x_{0, i}$, satisfying (2) and (3). We model the intersection as a closed and compact subset of positions along the path of each system, defined by the lower and upper bounds $L_{i}$ and $H_{i}$, respectively, as depicted in Fig. 1b. A vehicle is therefore inside the intersection at time $t$ if $x_{i}(t) \in \mathcal{E}_{i}=\left\{x \mid L_{i} \leq C_{i} x \leq H_{i}\right\}$, and the collision avoidance requirements are consequently

$$
\left[x_{i}^{T}(t), x_{j}^{T}(t)\right]^{T} \notin \mathcal{E}_{i} \times \mathcal{E}_{j}, \forall t, \forall i, j \in \mathcal{N}, i \neq j,
$$

where $\times$ denotes the cartesian product and $\mathcal{N}=\{1, \ldots, N\}$. Note that with a proper choice of $L_{i}$ and $H_{j}$ and $H_{i}-L_{i}$ large enough, we can in this way incorporate the vehicle geometry.

\section{A. Optimal Control Formulation}

Consider the local performance criterion

$$
J_{i}\left(x_{i}(t), u_{i}(t)\right)=\int_{0}^{t_{f}} \Lambda_{i}\left(x_{i}(t), u_{i}(t)\right) \mathrm{d} t
$$

where $\Lambda_{i}\left(x_{i}(t), u_{i}(t)\right)$ is quadratic and convex in $x_{i}(t)$ and $u_{i}(t)$, and $t_{f}$ is the final time. The problem of finding the optimal, physically feasible control functions that avoids collision can then be formalized as follows:
Problem 1 (Optimal Coordination Problem (OCP)). Given the initial states $x_{i}(0), i \in \mathcal{N}$ solve the problem

$$
\begin{aligned}
\min _{\mathbf{x}(t), \mathbf{u}(t)} & \sum_{i=1}^{N} J_{i}\left(x_{i}(t), u_{i}(t)\right) \\
\text { s.t. } & {\left[x_{i}^{T}(t), u_{i}^{T}(t)\right] \in D_{i}\left(x_{i}(0)\right), \forall i \in \mathcal{N} } \\
& {\left[x_{i}(t), x_{j}(t)\right] \notin \mathcal{E}_{i} \times \mathcal{E}_{j}, \forall t, \forall i \neq j }
\end{aligned}
$$

where $\mathbf{x}(t)=\left[x_{1}^{T}(t), \ldots, x_{N}^{T}(t)\right]^{T}, \quad \mathbf{u}(t)=$ $\left[u_{1}^{T}(t), \ldots, u_{N}^{T}(t)\right]^{T}$.

It is emphasized that the collision avoidance condition (6c) renders the problem non-convex, as visualized in Fig. 2. More precisely, a solution to (6) contains the best of the $N$ !

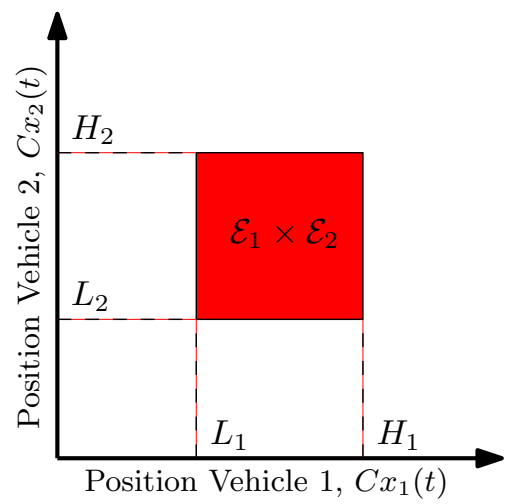

Fig. 2: Schematic illustration of a 2D cut of the state-space in (6). The red area contains the infeasible state space combinations according to (6c) and corresponds to collisions between vehicles 1 and 2 .

possible intersection crossing orders, in Fig. 2 corresponding to trajectories that goes above or below the red square. The problem is thus combinatorial and the solution need to be calculated using combinatorial optimization techniques.

In the next section, a decomposition is presented for the OCP, with the objective of designing low complexity algorithms for approximate solution.

\section{PROBLEM DECOMPOSITION}

In order to present the decomposition of Problem 1 we need to introduce the following additional notation. Given $C x_{i}(0)<L_{i}$, the time instance when the $i$-th vehicle enters $\left(\tau_{i}\right)$ and exits $\left(\xi_{i}\right)$ the intersection are defined as

$$
\tau_{i}=t: C_{i} x_{i}(t)=L_{i}, \quad \xi_{i}=t: C_{i} x_{i}(t)=H_{i}
$$

Note that (3) implies the uniqueness of the pair $\left(\tau_{i}, \xi_{i}\right)$ for a given $x_{i}(t)$, and that the occupancy time interval $\left[\tau_{i}, \xi_{i}\right]$, i.e., $t \in\left[\tau_{i}, \xi_{i}\right] \Leftrightarrow x_{i}(t) \in \mathcal{E}_{i}$, is closed and compact when $H_{i}>L_{i}$. With (7), condition (4) can therefore equivalently be restated as

$$
\left[\tau_{i}, \xi_{i}\right] \cap\left[\tau_{j}, \xi_{j}\right]=\emptyset, \forall i, j \in \mathcal{N}, i \neq j
$$

We first introduce the coordination problem, which opti- 
mally allocates occupancy timeslots to each vehicle as

$$
\begin{aligned}
\min _{\mathbf{T}, \mathbf{E}} & \sum_{i=1}^{N} F_{i}\left(\tau_{i}, \xi_{i}\right) \\
\text { s.t. } & {\left[\tau_{i}, \xi_{i}\right] \cap\left[\tau_{j}, \xi_{j}\right]=\emptyset, \forall i, j \in \mathcal{N}, i \neq j, } \\
& {\left[\tau_{i}, \xi_{i}\right] \in \mathcal{S}_{i}\left(x_{0, i}\right) . }
\end{aligned}
$$

where $\mathbf{T}=\left[\tau_{1}, . ., \tau_{N}\right]^{T}, \mathbf{E}=\left[\xi_{1}, \ldots, \xi_{N}\right]^{T}$, while $F_{i}\left(\tau_{i}, \xi_{i}\right)$ and $\mathcal{S}_{i}\left(x_{0, i}\right)$ are the value function and the set of feasible parameters, respectively, of the following local, convex parametric optimization problems

$$
\begin{array}{cl}
F_{i}\left(\tau_{i}, \xi_{i}\right)=\min _{x_{i}(t), u_{i}(t)} & J_{i}\left(x_{i}(t), u_{i}(t)\right) \\
\text { s.t. } \quad & {\left[x_{i}^{T}(t), u_{i}^{T}(t)\right] \in D_{i}\left(x_{0, i}\right),} \\
& C_{i} x_{i}\left(\tau_{i}\right)=L_{i}, \\
& C_{i} x_{i}\left(\xi_{i}\right)=H_{i},
\end{array}
$$

The solution to the right hand side of (9b) is thus the optimal input and state trajectories given the parameters $\tau_{i}$ and $\xi_{i}$, which has the cost $F_{i}\left(\tau_{i}, \xi_{i}\right)$. The following results then hold, where the proofs are reported in [12]:

Theorem 1. The optimization problems (6) and (9) are equivalent.

Furthermore, assuming $C x_{0, i}<L_{i}<H_{i}$ and $t_{f}$ sufficiently large, we define:

Definition 1 (Earliest and latest entry and exit times). The earliest (latest) entry time, $T_{i}^{l}\left(T_{i}^{h}\right) \in \mathbb{R}$ is defined as $\tau$ : $C_{i} x_{i}(\tau)=L_{i}$, where $x_{i}(t)$ is the solution to

$$
\begin{array}{cl}
\underset{x_{i}(t), u_{i}(t)}{\max (\min )} & C x\left(t_{f}\right) \\
\text { s.t. } & {\left[x_{i}^{T}(t), u_{i}^{T}(t)\right] \in D_{i}\left(x_{0, i}\right) .}
\end{array}
$$

Similarly, given $\tau_{i} \in\left[T_{i}^{l}, T_{i}^{h}\right]$, the earliest (latest) exit time, $E_{i}^{l}\left(\tau_{i}\right)\left(E_{i}^{h}\left(\tau_{i}\right)\right)$, is defined as $t: C_{i} x_{i}(t)=H_{i}$, where $x_{i}(t)$ is the solution to (10) with the additional constraint $C_{i} x_{i}\left(\tau_{i}\right)=L_{i}$.

The following result holds for the exit times:

Proposition 1. $E_{i}^{l}\left(\tau_{i}\right)$, and $E_{i}^{h}\left(\tau_{i}\right)$ are continuous, strictly increasing in $\left[T_{i}^{l}, T_{i}^{h}\right]$.

The sets $\mathcal{S}_{i}\left(x_{0, i}\right)$ of feasible parameters are then such that

Proposition 2. $\mathcal{S}_{i}\left(x_{0, i}\right)=\left\{\left(\tau_{i}, \xi_{i}\right): \tau_{i} \in\left[T_{i}^{l}, T_{i}^{h}\right]\right.$ and $\xi_{i} \in$ $\left.\left[E_{i}^{l}\left(\tau_{i}\right), E_{i}^{h}\left(\tau_{i}\right)\right]\right\}$ and is a closed and compact set.

Additionally, we define

Definition 2 (Optimal exit time). The optimal exit time given an entrance time $\tau_{i} \in\left[T_{i}^{l}, T_{i}^{h}\right]$ is defined as $g_{i}\left(\tau_{i}\right)=t$ : $C_{i} x_{i}(t)=H_{i}$, where $x_{i}(t)$ is the minimizer of

$$
\begin{array}{cl}
\min _{x_{i}(t), u_{i}(t)} & J_{i}\left(x_{i}(t), u_{i}(t)\right) \\
\text { s.t. } & {\left[x_{i}^{T}(t), u_{i}^{T}(t)\right] \in D_{i}\left(x_{0, i}\right),} \\
& C_{i} x_{i}\left(\tau_{i}\right)=L_{i} .
\end{array}
$$

It immediately follows that $g_{i}\left(\tau_{i}\right)$ is uniquely defined (due to convexity of (11)), continuous and that $E_{i}^{l}\left(\tau_{i}\right) \leq g_{i}\left(\tau_{i}\right) \leq$

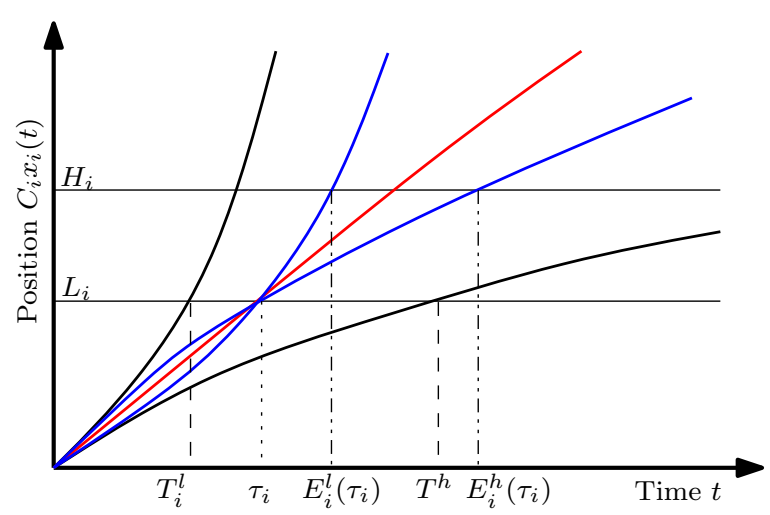

Fig. 3: Schematic visualization of the defining elements of $\mathcal{S}_{i}\left(x_{0, i}\right)$. The curved black lines represent the trajectories resulting from maximum and minimum control signal respectively. The curved blue lines show the same given that the entrance time $\tau_{i}$ is enforced. The red line is the optimal trajectory when $\tau_{i}$ is enforced.

$E_{i}^{h}\left(\tau_{i}\right)$. It also follows that $F_{i}\left(\tau_{i}, g_{i}\left(\tau_{i}\right)\right) \leq F_{i}\left(\tau_{i}, \xi_{i}\right)$, with equality only for $\xi_{i}=g_{i}\left(\tau_{i}\right)$, and that the minimizer $\left[\tau_{i}^{*}, \xi_{i}^{*}\right]$ of $F_{i}\left(\tau_{i}, \xi_{i}\right)$ is such that $g_{i}\left(\tau_{i}^{*}\right)=\xi_{i}^{*}$. We then have:

Theorem 2. Solutions to (9a) satisfy $\xi_{i} \leq g_{i}\left(\tau_{i}\right), i \in \mathcal{N}$.

Proposition 3. $F_{i}\left(\tau_{i}, \xi_{i}\right)$ has a unique minimum in $\mathcal{S}_{i}\left(x_{0, i}\right)$.

Proposition 4. $F_{i}\left(\tau_{i}, \xi_{i}\right)$ is increasing with $\left|\tau_{i}-\tau_{i}^{*}\right|$ and $\left|\xi_{i}-\xi_{i}^{*}\right|$.

To summarize, $\mathcal{S}_{i}\left(x_{0, i}\right)$ is a closed and compact set in the $\left[\tau_{i}, \xi_{i}\right]$ parameter space, implicitly defined through the constraints (1), (2) and (3), and $F_{i}\left(\tau_{i}, \xi_{i}\right)$ is "bowl-shaped" with a unique minimum over this set.

\section{Approximation}

In this section, the results presented in Section III will be used to construct a computational scheme that approximately solves (6). More precisely, we propose a two staged procedure where $(9 \mathrm{a})$ first is solved using approximations of $F_{i}\left(\tau_{i}, \xi_{i}\right)$ and $\mathcal{S}_{i}\left(x_{0, i}\right)$ for approximately optimal occupancy time slots $\left[\tau_{i}^{*}, \xi_{i}^{*}\right]$. Using these, a relaxation of $(9 \mathrm{~b})$ is then solved for each vehicle to obtain the state and control trajectories $x_{i}^{*}(t)$ and $u_{i}^{*}(t)$. Conditions are given on how $F_{i}\left(\tau_{i}, \xi_{i}\right)$ and $\mathcal{S}_{i}\left(x_{0, i}\right)$ must be formed to guarantee that a solution to the approximation scheme is feasible in terms of (6).

\section{A. Relaxation}

Consider the following relaxation of problem (9b)

$$
\begin{array}{cl}
\min _{x_{i}(t), u_{i}(t)} & J_{i}\left(x_{i}(t), u_{i}(t)\right) \\
\text { s.t. } & {\left[x_{i}^{T}(t), u_{i}^{T}(t)\right] \in D\left(x_{0, i}\right),} \\
& C x\left(\tau_{i}^{*}\right) \leq L_{i}, \\
& C x\left(\xi_{i}^{*}\right) \geq H_{i},
\end{array}
$$

and denote by $x_{i}^{*}(t), u_{i}^{*}(t)$ its solution for a given $\left[\tau_{i}, \xi_{i}\right]$. The actual entry and exit time $\hat{\tau}_{i}=t: C_{i} x_{i}^{*}(t)=L_{i}, \hat{\xi}_{i}=$ $t: C_{i} x_{i}^{*}(t)=H_{i}$ are then such that $\left[\hat{\tau}_{i}, \hat{\xi}_{i}\right] \subseteq\left[\tau_{i}, \xi_{i}\right]$, since by (3), $C x_{i}^{*}\left(\tau_{i}\right)<L_{i} \Rightarrow \hat{\tau}_{i}>\tau_{i}$ and $C x_{i}^{*}\left(\xi_{i}\right)>H_{i} \Rightarrow \hat{\xi}_{i}<\xi_{i}$. 
Note that due to (3), solutions exist to (12) provided that (i) $\tau_{i} \leq T_{i}^{h}$ since otherwise $\hat{\tau}_{i}>T_{i}^{h}$, and (ii) $\xi_{i}^{*} \geq E_{i}^{l}\left(\tau_{i}\right)$, since otherwise $\hat{\xi}_{i} \geq E_{i}^{l}\left(\tau_{i}\right)$. The bounds $\tau \geq T_{i}^{l}$ and $\xi \leq E_{i}^{h}\left(\tau_{i}\right)$ on the other hand, do not affect the feasibility of (12) due to the direction of the inequalities (12c) and (12d).

\section{B. Explicit approximation of $\mathcal{S}_{i}\left(x_{0, i}\right)$}

We first note that the solution to (9a) are sought in $\mathcal{S}_{i}^{e}\left(x_{0, i}\right)=\mathcal{S}_{i}\left(x_{0, i}\right) \backslash\left\{\tau, \xi \mid \xi>g_{i}(\tau)\right\}, i=1, \ldots, N$, according to Theorem 2 . Due to this it is only necessary to find an approximation $\hat{\mathcal{S}}_{i}^{e}\left(x_{0, i}\right)$ of $\mathcal{S}_{i}^{e}\left(x_{0, i}\right)$. To ensure that all elements in the approximation are feasible in terms of the relaxed local problem, it is as described above necessary that all $\left[\tau_{i}, \xi_{i}\right] \in \hat{\mathcal{S}}_{i}^{e}$ satisfy $\tau \leq T_{i}^{h}$ and $E_{i}^{l}\left(\tau_{i}\right) \leq \xi_{i}$ for problem (12). Similarly, to avoid removing the optimal exit time given $\tau_{i}, g_{i}\left(\tau_{i}\right), \hat{\mathcal{S}}_{i}^{e}\left(x_{0, i}\right)$ must be such that $\xi_{i} \leq$ $g_{i}(\tau)$. While $T_{i}^{l}, T_{i}^{h}$ are easily computed through Definition 1 , neither $E_{i}^{l}\left(\tau_{i}\right)$ nor $g_{i}\left(\tau_{i}\right)$ are easily obtained. However, by choosing strictly increasing functions $u_{i}\left(\tau_{i}\right)$ and $l_{i}\left(\tau_{i}\right)$ such that $g_{i}\left(\tau_{i}\right) \leq u_{i}\left(\tau_{i}\right), E_{i}^{l}\left(\tau_{i}\right) \leq l_{i}\left(\tau_{i}\right)$ and $l_{i}\left(\tau_{i}\right) \leq$ $u_{i}\left(\tau_{i}\right), \forall \tau_{i} \in\left[T_{i}^{l}, T_{i}^{h}\right]$ and letting

$$
\hat{\mathcal{S}}_{i}^{e}\left(x_{0, i}\right)=\left\{\tau_{i}, \xi_{i} \mid \tau_{i} \in\left[T_{i}^{l}, T_{i}^{h}\right], \xi_{i} \in\left[l_{i}(\tau), u_{i}(\tau)\right]\right\},
$$

we have by Proposition 2 that (12) is feasible $\forall\left[\tau_{i}, \xi_{i}\right] \in$ $\hat{\mathcal{S}}_{i}^{e}$, and that $\left[\tau_{i}, g_{i}\left(\tau_{i}\right)\right] \in \hat{\mathcal{S}}_{i}^{e}\left(x_{0, i}\right), \forall \tau_{i} \in\left[T_{i}^{l}, T_{i}^{h}\right]$, without direct use of $g_{i}\left(\tau_{i}\right)$ or $E_{i}^{l}\left(\tau_{i}\right)$. Consequently, if occupancy times $\left[\tau_{i}, \xi_{i}\right] \in \hat{\mathcal{S}}_{i}^{e}\left(x_{0, i}\right), i=1, \ldots, N$ are such that $\left[\tau_{i}, \xi_{i}\right] \cap$ $\left[\tau_{j}, \xi_{j}\right]=\emptyset$, then the actual occupancy times $\left[\hat{\tau}_{i}, \hat{\xi}_{i}\right]$, are such that $\left[\hat{\tau}_{i}, \hat{\xi}_{i}\right] \cap\left[\hat{\tau}_{j}, \hat{\xi}_{j}\right]=\emptyset$ and therefore also feasible in (9). The solutions $\left[x_{i}^{*}(t), u_{i}^{*}(t)\right]$ to (12) for $i=1, \ldots, N$ corresponding to $\left[\hat{\tau}_{i}, \hat{\xi}_{i}\right]$, are in that case also feasible in (6) according to Theorem 1 .

Remark 1. Note that usage of this approximation has two consequences. First, all $\left[\tau_{i}, \xi_{i}\right] \in \mathcal{S}_{i}^{e}\left(x_{0, i}\right) \backslash \hat{\mathcal{S}}_{i}^{e}\left(x_{0, i}\right)$ are feasible in the exact formulation of $(9 \mathrm{a})$, but a priori removed from the approximate formulation . Second, all $\left[\tau_{i}, \xi_{i}\right] \in$ $\hat{\mathcal{S}}_{i}^{e}\left(x_{0, i}\right) \backslash \mathcal{S}_{i}^{e}\left(x_{0, i}\right)$ could be solutions to the approximate formulation of $(9 \mathrm{a})$, but by Theorem 2 never to the exact one. Consequently, the scheme is conservative as larger timeslots than strictly needed might be retrieved from the solution of the approximate formulation. Tighter bounding functions $l_{i}(t)$ and $u_{i}(t)$ will reduce the conservativeness and expand the set of feasible solutions. An illustration of the set $\mathcal{S}_{i}\left(x_{0, i}\right)^{e}$ and its approximation $\hat{\mathcal{S}}_{i}^{e}$ based on linear bounds $l_{i}\left(\tau_{i}\right), u_{i}\left(\tau_{i}\right)$ is given in Fig. 4.

\section{Explicit approximation of $F_{i}\left(\tau_{i}, \xi_{i}\right)$}

According to Propositions 3 and $4, F_{i}\left(\tau_{i}, \xi_{i}\right)$ has a unique minimum at $\tau_{i}^{*}, \xi_{i}^{*}$ and increases with $\left|\tau_{i}-\tau_{i}^{*}\right|$ and $\left|\xi_{i}-\xi_{i}^{*}\right|$. We therefore propose the use of a strictly convex function $\hat{F}_{i}\left(\tau_{i}, \xi_{i}\right)$ with minimum in $\tau_{i}^{*}, \xi_{i}^{*}$.

\section{Proposed Algorithm}

The three main steps of the proposed coordination algorithms are summarized as follows:

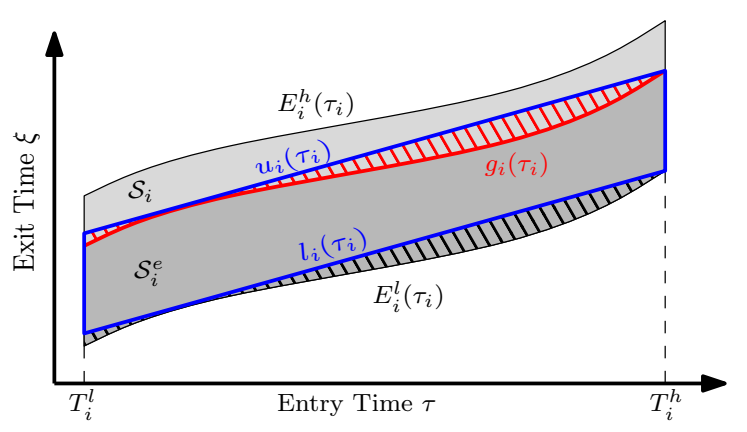

Fig. 4: Schematic visualization of the proposed approximation method for $\mathcal{S}_{i}\left(x_{0, i}\right)$. The blue bordered area represents $\hat{\mathcal{S}}_{i}^{e}\left(x_{0, i}\right)$, the black bordered area $\mathcal{S}_{i}\left(x_{0, i}\right)$. The area in dashed black shows $\mathcal{S}_{i}^{e}\left(x_{0, i}\right) \backslash \hat{\mathcal{S}}_{i}^{e}\left(x_{0, i}\right)$, i.e., feasible solutions that are lost in the approximation, whereas $\hat{\mathcal{S}}_{i}^{e}\left(x_{0, i}\right) \backslash$ $\mathcal{S}_{i}^{e}\left(x_{0, i}\right)$, shown in dashed red, contains elements that are unnecessarily considered.

1) [Offline] $\forall i \in \mathcal{N}:$ Compute $\hat{\mathcal{S}}_{i}^{e}\left(x_{0, i}\right)$ and $\hat{F}_{i}\left(\tau_{i}, \xi_{i}\right)$. The calculation can be independently performed by each vehicle.

2) [Online] Centrally solve (9a) with $\hat{\mathcal{S}}_{i}^{e}\left(x_{0, i}\right)$ and $\hat{F}_{i}\left(\tau_{i}, \xi_{i}\right)$ calculated at step 1 , to obtain $\left[\tau_{i}^{*}, \xi_{i}^{*}\right], \forall i \in$ $\mathcal{N}$. If not solvable, return infeasible.

3) [Online] $\forall i \in \mathcal{N}$ : With $\left[\tau_{i}^{*}, \xi_{i}^{*}\right]$, solve (12) for minimizers $x_{i}^{*}(t), u_{i}^{*}(t), \forall i \in \mathcal{N}$.

Due to the construction of $\hat{\mathcal{S}}_{i}^{e}\left(x_{0, i}\right)$, the algorithm will in general be conservative as described in Remark 1. In particular, Problem (9a) might be infeasible with $\hat{\mathcal{S}}_{i}^{e}\left(x_{0, i}\right)$, although feasible with $\mathcal{S}_{i}^{e}\left(x_{0, i}\right)$. However, since (12) is feasible for all solutions to the approximate formulation of (9a), the algorithm cannot provide a solution that is infeasible in (6).

\section{NuMERICAL EXAMPLE}

In this section, we present a numerical evaluation of the algorithm introduced in Section IV and details the three steps described in Section IV-D. Results from an evaluation over a range of random vehicle configurations is presented. In particular, the performance of the proposed algorithms is compared against the exact solution in terms of suboptimality and effective execution time (using commercially available standard solvers) is presented and discussed.

\section{A. Vehicle Model}

Problem (6) is discretized on a uniform time grid of size $K \in \mathbb{R}_{+}$, so that the sample time $\Delta t=t_{f} / K$ Furthermore, the vehicles are modelled as double integrators with the state vector is defined as $x_{i}(k)=\left[\begin{array}{ll}p_{i}(k) & v_{i}(k)\end{array}\right]^{T}$. Here $p_{i}(k), v_{i}(k)$ are position and velocity of the vehicle along the path, respectively, and $k$ is the discrete time index, while the control $u_{i}(k)$ is the vehicle acceleration. The constraints (2) and (3) are chosen as $\underline{u}_{i} \leq u_{i}(k) \leq \bar{u}_{i}$ and $\quad 0<$ $\epsilon_{i} \leq v_{i}(k)$ respectively. Further, we let $\Lambda_{i}\left(x_{i}(k), u_{i}(k)\right)=$ $\left(v_{d, i}-v_{i}(k)\right)^{2} Q_{i}+u_{i}^{2}(k-1) R_{i}$ in (5), where $v_{d, i} \in \mathbb{R}_{+}$is a constant reference velocity and $Q_{i}, R_{i} \in \mathbb{R}_{+}$. For simplicity, the intersection is defined equally large for all vehicles, i.e., so that $H_{i}-L_{i}=H_{j}-L_{j}, \forall i, j \in \mathcal{N}$. 


\section{B. Exact Solution}

The constraints (6c) are enforced by introducing auxiliary binary decision variables, $\delta_{i}(k), \gamma_{i}(k) \in\{0,1\}$, and for $i=$ $1, \ldots, N$ and $k=1, \ldots, N$ require that

$$
\begin{array}{r}
C_{i} x_{i}(k) \leq L_{i}+\delta_{i}(k) M, \\
H_{i}-\gamma_{i}(k) M \leq C_{i} x_{i}(k), \\
\delta_{i}(k)+\delta_{j}(k)+\gamma_{i}(k)+\gamma_{j}(k) \leq 3,
\end{array}
$$

where $M$ is a sufficiently large number. The resulting problem is a mixed binary integer quadratic program (MBIQP) with $2 N K$ binary and $\sum_{i=1}^{N} m_{i}+n_{i}$ continuous variables, with $K \sum_{i=1}^{N} n_{i}$ equality and $K \sum_{i=1}^{N}\left(q_{i}+k_{i}+1\right)+2 N(N-$ 1) inequality constraints.

\section{Approximate Solution}

With the purpose of stating also the approximate formulation of (9a) as a MBIQP, the bounding functions $l_{i}\left(\tau_{i}\right)$ and $u_{i}\left(\tau_{i}\right)$ are chosen affine, and the approximation $\hat{F}_{i}\left(\tau_{i}, \xi_{i}\right)$ quadratic.

Step 1: The earliest and latest entry times $\left[T_{i}^{l}, T_{i}^{h}\right]$ are first obtained through solution of (10). Samples of $E_{i}^{l}\left(\tau_{i}\right)$ and $g_{i}\left(\tau_{i}\right)$ on a grid of $\tau_{i} \in\left[T_{i}^{l}, T_{i}^{h}\right]$ are then obtained directly through Definition 1 and Definition 2. Affine functions $l_{i}\left(\tau_{i}\right)$ and $u_{i}\left(\tau_{i}\right)$ are thereafter fitted to the data, constrained to satisfy $E_{i}^{l}\left(\tau_{i}\right) \leq l_{i}\left(\tau_{i}\right) \leq u_{i}\left(\tau_{i}\right)$ and $u_{i}\left(\tau_{i}\right) \geq$ $g_{i}\left(\tau_{i}\right)$ at all sampled $\tau_{i}$, giving the required components of $\hat{\mathcal{S}}_{i}^{e}\left(x_{0, i}\right)$. Similarly, samples of $F_{i}\left(\tau_{i}, \xi_{i}\right)$ are obtained by solving (9b) on a grid of $\left[\tau_{i}, \xi_{i}\right] \in \hat{\mathcal{S}}_{i}^{e}\left(x_{0, i}\right)$, after which a quadratic form $\hat{F}_{i}\left(\tau_{i}, \xi_{i}\right)$ is fitted to the data. The retrieval of the samples involves solving multiple LP's, $\left.E_{i}^{l}\left(\tau_{i}\right)\right)$ and QP's $\left(g_{i}\left(\tau_{i}\right), \hat{F}_{i}\left(\tau_{i}, \xi_{i}\right)\right.$, whereas the function fitting is done through LP's $\left(E_{i}^{l}\left(\tau_{i}\right), g_{i}\left(\tau_{i}\right)\right)$ and SDP's $\left(\hat{F}_{i}\left(\tau_{i}, \xi_{i}\right)\right)$.

Step 2: Using the computed approximations, (9a) is solved as a MBIQP, where $\left[\tau_{i}, \xi_{i}\right] \cap\left[\tau_{j}, \xi_{j}\right]=\emptyset$ is enforced through $\xi_{i} \leq \tau_{j}+M \delta_{i j}$, and $\xi_{j} \leq \tau_{i}+M\left(1-\delta_{i j}\right)$, where $\delta_{i j}, i \neq j$, are binary decision variables and $M$ is a large number. This problem has $2 N$ continuous decision variables, $1 / 2(N-1) N$ binary decision variables and $N^{2}+3 N$ affine inequality constraints.

Step 3: With the non-overlapping occupancy timeslots from Step 2, (12) is solved for each of the $N$ vehicles.

\section{Simulation set-up}

The evaluation is carried out on a class of scenarios with six vehicles, where for each vehicle the initial conditions $x_{0}$, desired speed $v_{d}[\mathrm{~m} / \mathrm{s}]$, actuation limits $\underline{u}, \bar{u}\left[\mathrm{~m} / \mathrm{s}^{2}\right]$ and objective function weights $Q, R$, are all drawn from the uniform distribution on the ranges given in Table I. Other relevant parameters that are set to $H_{i}-L i=10[\mathrm{~m}]$, $K=100, \Delta t=0.1[\mathrm{~s}], \varepsilon>0.01[\mathrm{~m} / \mathrm{s}]$. Given a scenario instance, the exact problem (6) is solved first, whereafter the approximate algorithm runs only if (i) a feasible solution to (6) exists, and (ii) the solution is non-trivial (i.e. the solution requires some adaptation to avoid collisions). The linear programs of Step 1 and quadratic programs of Steps 1 and 3, as well as the MIQPB's (6), (9a), are solved using CPLEX.

\begin{tabular}{|c|c|c|c|c|c|c|c|}
\hline & $p_{0}$ & $v_{0}$ & $\underline{u}$ & $\bar{u}$ & $v_{d}$ & $Q$ & $R$ \\
\hline $\min$ & -100 & 30 & -3 & 1 & 30 & 1 & 1 \\
\hline $\max$ & -50 & 90 & -1 & 3 & 90 & 10 & 10 \\
\hline
\end{tabular}

TABLE I: Ranges for the vehicles parameters in the simulation. Note that $x_{0}=\left[p_{0}, v_{0}\right]^{T}$.

\section{E. Results}

The following results were obtained from 1000 instances drawn from the scenario envelope. The running time performance recorded is given in Table II, where the comparison is made between the parts of the solutions that by necessity must be at least coordinated centrally, i.e., the entire (6) and (9a) for the exact and approximate solutions respectively. The proposed algorithms performance in terms of sub-optimality is shown in Fig. 5, computed as $\left(\hat{J}^{*}-J^{*}\right) / J^{*}$, where $J^{*}$ and $\hat{J}^{*}$ is the cost of the exact and approximate solution respectively. Furthermore, it is noted that in 9 realizations $(\approx$ $1 \%$ ), a feasible solution existed to (6) but not to (9a), showing the conservativeness introduced by the approximation as discussed in Remark 1. An example of the resulting timeposition trajectories is given in Fig. 6.

\begin{tabular}{|c|c|c|}
\hline & Mean & $s$ \\
\hline Exact solution, (6) & $10.14[\mathrm{~s}]$ & $24.067[s]$ \\
\hline Approximation, Step 2, (9a) & $0.043[\mathrm{~s}]$ & $0.022[s]$ \\
\hline
\end{tabular}

TABLE II: Statistics on time performance as reported by MIQPB solver over the examined 1000 instances, where $s$ is the empirical standard deviation. The compared MBIQP's (6) and (9a) are solved with CPLEX on a $1.9 \mathrm{GHz}$ Intel i5 desktop with 8 GB RAM, running Windows 7.

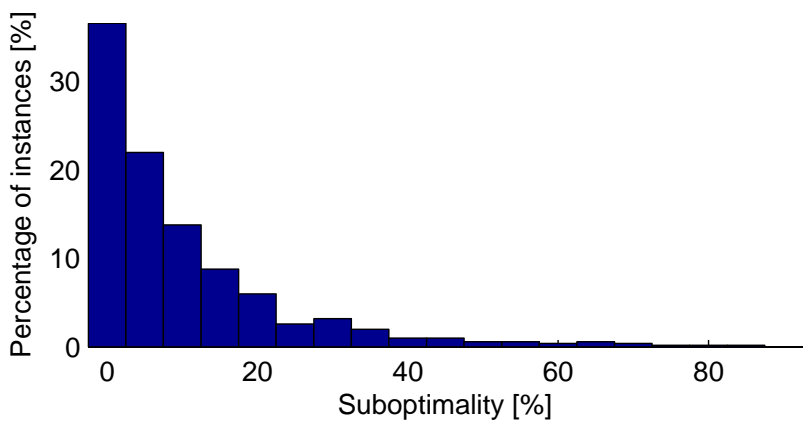

Fig. 5: Distribution of the performance of the proposed algorithm in terms of sub-optimality for 1000 instances drawn from the scenario envelope with parameters according to Table I

\section{DISCUSSION}

Although both (6) and the proposed approximation scheme has exponential worst time complexity, the difference in practice is clearly substantial (c.f. Table II). This is a natural consequence of the large decrease in problem size from (6) to (9a). Note in particular that neither the dynamics nor the discrete time horizon has any effect on the size of the actual 


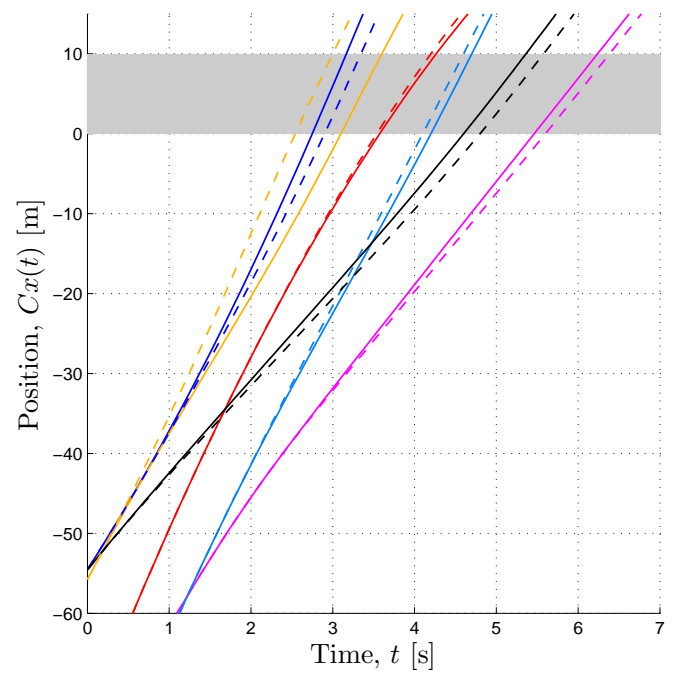

Fig. 6: Time-position plot of one realization from the envelope of scenarios described with parameters according to Table I. The grey band represents the intersection (equally large for all vehicles) and the different coloured trajectories the position of the different vehicles, where solid lines correspond to exact solutions and dashed lines to approximate. Note in particular the dark blue and orange trajectories, that shows that the approximate solution has found a different crossing order than the direct.

coordination problem, enabling the use of arbitrary large models and horizons. The computational effort is instead largely moved to Steps 1 and 3 of the approximation procedure, which can be computed a-priori and retrieved from memory, or in parallel (i.e. on-board the different vehicles). The price paid is sub-optimality, which is directly dependent on the size of $\hat{\mathcal{S}}_{i}^{e}\left(x_{0, i}\right) \backslash \mathcal{S}_{i}^{e}\left(x_{0, i}\right), \mathcal{S}_{i}^{e}\left(x_{0, i}\right) \backslash \hat{\mathcal{S}}_{i}^{e}\left(x_{0, i}\right)$, and on the quality of the $\hat{F}_{i}\left(\tau_{i}, \xi_{i}\right)$ fit. Consequently, other choices of e.g. $l_{i}\left(\tau_{i}\right), u_{i}\left(\tau_{i}, \xi_{i}\right)$ than affine functions could increase the performance of the approximation. However, it is worth emphasizing that even though the approximations are constructed with simple functions, the algorithm gives results below $20 \%$ suboptimality in around $85 \%$ of the realizations. Furthermore, the proposed method offers a natural coding of the basic components of cooperative decision making; the options of each participant $\left(\hat{\mathcal{S}}_{i}^{e}\left(x_{0, i}\right)\right)$ and the associated preferences $\left(\hat{F}_{i}\left(\tau_{i}, \xi_{i}\right)\right)$. The compact representation has beneficial consequences also for the design and evaluation of the associated communication system, as the information exchange is small and performance requirements can be derived easily. Finally, the set $\mathcal{S}_{i}\left(x_{0, i}\right)$ and cost function $F_{i}\left(\tau_{i}, \xi_{i}\right)$ presented in this paper can be viewed as the result of a multi parametric program (MPP). Although a rich theory exists for standard linear and quadratic MPP's, problem (9b) differs fundamentally in that the parameters $\left[\tau_{i}, \xi_{i}\right]$ enters the formulation in a non-standard fashion.

\section{CONCLUSIONS}

In this paper, we have presented an algorithm for approximate solution to the intersection problem for autonomous vehicles. In our algorithm, the problem is parametrized with the intersection entry and exit times, and given a hierarchical structure with a central optimization-based coordinator. The influence of the individual vehicle dynamics are condensed and approximatively represented with simple expressions. The main benefits of the presented scheme are: (i) the nearoptimality and dynamic feasibility of the obtained solutions, (ii) the ability to use objectives conditioned on the individual vehicle states (energy usage etc.), (iii) a significant reduction of the computational demands on the central unit, and (iv) a low and predictable demand on the communication system, resulting from the compact representation of each vehicles possibilities and preferences. In future work, we intend to investigate the closed loop behaviour of the proposed algorithm, the influence of communication related uncertainties (packet drops etc.) as well as further reductions in complexity.

\section{REFERENCES}

[1] H. Wymeersch, G. R. Campos, P. Falcone, L. Svensson, and E. Ström, "Challenges for cooperative its: Improving road safety through the integration of wireless communications, control, and positioning," in Proc. of the International Conference on Computing, Networking and Communications (ICNC), 2015.

[2] M. Simon, T. Hermitte, and Y. Page, "Intersection road accident causation: A European view," Proc. of the 21st International Technical Conference on the Enhanced Safety of Vehicles, pp. 1-10, 2009.

[3] E. Steinmetz, R. Hult, G. Campos, P. Falcone, and H. Wymeersch, "Communication analysis for centralized intersection crossing coordination," in Proc. of the Symposium on Wireless Communication Systems (ISWCS), 2014.

[4] K. Dresner and P. Stone, "Multiagent traffic management: a reservation-based intersection control mechanism," in Proc. of the Third International Joint Conference on Autonomous Agents and Multiagent Systems, pp. 530-537, 2004.

[5] H. Kowshik, D. Caveney, and P. Kumar, "Provable systemwide safety in intelligent intersections," IEEE Transactions on Vehicular Technology, vol. 60, no. 3, pp. 804-818, 2011.

[6] J. Lee and B. Park, "Development and evaluation of a cooperative vehicle intersection control algorithm under the connected vehicles environment," IEEE Transactions on Intelligent Transportation Systems, vol. 13, no. 1, pp. 81-90, 2012.

[7] A. Colombo and D. Del Vecchio, "Efficient algorithms for collision avoidance at intersections," in Proc. of the 15th ACM International Conference on Hybrid Systems: Computation and Control, 2012.

[8] K.-D. Kim and P. Kumar, "An MPC-based approach to provable system-wide safety and liveness of autonomous ground traffic," IEEE Transactions on Automatic Control, vol. 59, no. 12, pp. 3341-3356, 2014.

[9] G. R. Campos, P. Falcone, and J. Sjöberg, "Autonomous cooperative driving: a velocity-based negotiation approach for intersection crossing," in Proc. of the IEEE Conference on Inteligent Transportation Systems, 2013.

[10] G. R. Campos, P. Falcone, H. Wymeersch, R. Hult, and J. Sjöberg, "A receding horizon control strategy for cooperative conflict resolution at traffic intersections," in Proc. of the IEEE Conference on Decision and Control (CDC), 2014.

[11] S. R. Azimi, G. Bhatia, and R. R. Rajkumar, "Reliable intersection protocols using vehicular networks," ACM/IEEE 4th International Conference on Cyber-Physical Systems (ICCPS), 2013.

[12] R. Hult, G. R. Campos, P. Falcone, and H. Wymeersch, "Technical report: Approximate solution to the optimal coordination problem for autonomous vehicles at intersections," tech. rep., Department of Signals and Systems, Chalmers University of Technology, avaliable at http://www.gabrieldecampos.com/Documents / Publications/Technical_Report_ACC15_safe.pdf, Mars 2015. 